\title{
The application of ontology in the machinery industry
}

\author{
Jinwen Li, Jiali Zhang, Lijian Hao \\ Chinese North Engine Research Institute
}

Keywords: Ontology, Knowledge expression, Product information model, Mechanical Engineering.

\begin{abstract}
Research on Ontology has been widely applied in the various walks of life, but in the machinery industry it's comparatively rare. Therefore, it is very necessary to carry on the overview of the application of ontology in mechanical industry. Firstly, this paper introduces the basic concept of ontology and related content. Secondly, all kinds of introduction of the application of ontology in the machinery industry are introduced. Finally, through the analysis of ontology application in the machinery industry, it's necessary to raise the application framework and the prospect of ontology in mechanical industry.
\end{abstract}

\section{Introduction}

In 1993, Professor Gruber of the Stanford University Knowledge Systems Laboratory first proposed the Ontology theory [1].It is a new beginning for this philosophy concept of Ontology into the field of computer. Ontology literally translated into "ontology is an illustration of the conceptual explanation".

At present, the construction of Ontology is regarded as improving the working process of the knowledge, strengthening the knowledge sharing and reusing and promoting the interoperation method of the heterogeneous system. The application of Ontology is very extensive including: the enterprise integration, the common knowledge, the semantic Web, the natural language translation, the mechanical engineering, the pharmaceutical engineering, the software engineering and the electronic commerce.

\section{The related concepts of ontology}

In the field of information, ontology as an expression of knowledge, including the object name in the field of research, object properties and mutual relationships, and so on, provides the knowledge exchange in the field and collection of expression, as well as the relationship collection of objects. In the 1990s, Neches and Lenat, respectively, in research projects—take the ontology references to the expression of knowledge, this method of ontology building played a revolutionary role.

\section{The meaning of ontology}

The core content of ontology definition is a conceptual model, that is: the object appears in the field and concepts and the link between them is the simply, an abstract view in the area of research. The main body of each level knowledge, knowledge base system and knowledge base all show or hide some of the conceptual model.

Classification is in accordance with the nature, characteristics and uses, etc. of things as distinguishing criteria, to meet the same standards of thing clustering, the different things that separate a standard way to understand things.

Compared with ontology and taxonomy, there are two main differences [2]: the design of objects is based on the operational attributes of the class; design of ontology is based on the structural properties of the class. Ontology can describe clearer object classes, objects and object hierarchy structure, and you can also describe other kinds of relationships between objects. Ontology not only describes the corresponding classification method, and a conceptual model for further restrictions. Need on the basis of classification reference axiom sets for constraint interpretation of the industry jargon. In theory, we can be based on the following five contents of the ontology to describe: concept, functions, axioms, relations and instances. The concept of ontology is refers to anything in their field. For example: the thing itself, the behavior of things, things function, the logical thing 
and so on. The function in the ontology refers to the relationship between internal based on ontology, through the logical inference has certain theory value of the formula are obtained. The axiom in the ontology refers to the related public rational explanation of the ontology. The relation between ontology refers to the restriction relationship between concepts. Examples of ontology refer to the characteristic of ontology concepts entity.

\section{The mathematical definition of ontology}

According to the University of Karlsruhe, Germany knowledge ontology working group research content, quintuple structure of ontology expressed as follows:

Definition: quintuple structure of ontology is as follows:

$$
O:=<C, \leq \mathrm{c}, R, \sigma, \leq R>
$$

Among them, $\mathrm{C}$ is the collection of concept, the elements in the $\mathrm{C}$ is the concept of markers; $\mathrm{R}$ represents the relationship, the elements in the $\mathrm{R}$ represent the relationship between the identifier; and $C \cap R=\varnothing$; partial order on the set $\mathrm{C}$ is $\leq \mathrm{c}$, called taxonomy or concept hierarchy, the signature is: $\sigma: R \rightarrow C^{+}$; is a partial order relation on the set $\mathrm{R}$, called hierarchy relationship, when $r_{1} \leq R r_{2}$, each $i: 1 \leq i \leq\left|\sigma\left(r_{1}\right)\right|$ has $\left|\sigma\left(r_{1}\right)\right|=\left|\sigma\left(r_{2}\right)\right|, \pi_{i}\left(\sigma\left(r_{1}\right)\right) \leq c \pi_{i}\left(\sigma\left(r_{2}\right)\right)$, the concept identifiers and relation identifier, respectively referred to as the concepts and relationships [3-8].

\section{classification principles of ontology}

Through the dependence of ontology on a single field, reflect generalization of the ontology, the lower the dependence on the field, the higher the level of generality ontology [9]. According to this principle, the ontology has the following classification:

- Domain Ontology: describe some domain knowledge.

- General ontology: general knowledge in many fields.

- Application ontology: in solving a particular problem, the need of knowledge.

- Says ontology: provides a set of application-independent knowledge to represent ontology,

- Task and method of ontology: description method of ontology to solve specific tasks.

\section{The methodology of ontology}

\section{The methodology}

Specific domain ontology construction is summed up in the field of property, concepts, relationships, constraints and axioms. Common ontology methodology includes: Uschold development skeleton method used by enterprise ontology, CYC ontology method, FOX and Gruninger in TOVE enterprises to establish a method of ontology, Lopez et al., Development CHENMICALS ontology employed Methnontology method, KACTUS project and Berneras methods, Protégé method and SENESUS ontology method. Which, Methnontology method, TOVE methods and skeleton method is relatively mature, they are all consider from the field of get areas and application of knowledge, after acquiring a lot of knowledge, TOVE methods and skeleton method uses the form of language coding. And Methnontology method is using middleware to form encoding. Protégé method is using iterative model, from course to fine, gradually break down demand, eventually form a unified planning of ontology. The implementation phase of the project, you can choose the different project methodology according to different characteristics [10-13].

\section{The application of ontology in mechanical industry}

\section{Ontology model of product information}

US National Institute of Standards and Technology in collaboration with NIST, in order to meet the information needs of the product lifecycle, the ontology model as a tool, developed this kind of Product information model framework. NIST products classified in the following three models: Desige Analytic Intergrated Model (DAIM), Open Assembly Model (OAM), PFEM. Among them, CPM describe the product form, behavior and function, as well as the relationship between these concepts. By CPM design principles can be combined with the product. OAM describes the conceptual model of hierarchical assembly relationships and systems [14]. DAIM functional model 
defines an abstract model of the main product, and the mutual relationship between them. PFEM represents the product family and related components; at the same time in the product family relationship is also included in it.

Product information model framework as a process of many aspects in the design of database, provide consistent information for PLM and other information exchange protocol, to support other system directly with CAX interoperability and seamless link between [15]. This framework has the following features:

- By using the appropriate ontology, and in formal language as the basis for automated reasoning.

- Has the characteristics of ontology, to physical domain concepts described (objects and attributes), rather than for a specific product (engine, etc.) is described.

- Multi-directional information integration.

- For new application development, alleviate design research and development process, the design research and development information is not complete hindered the implementation of new applications.

- The clear design principles embodied in the framework as a part of the product content.

- As an excuse for communicate with each other and switching between multiple systems modules.

\section{Semantic ontology}

In ISO10303 standard, in the process of product data exchange, grammar content does not include in the semantics of the related content, in order to compensate for this defect, the researchers on the study the information exchange of semantic expression given sufficient attention.

For product data semantics of each operational problem, University of Michigan related scholars have developed Product ontology in the most standard intermediary, and as a Semantic Representation Language (Product Semantic Representation Language PSRL) (16-19). Products semantic interoperability refers to the product development life cycle, the mutual flow of various information resources and can automatically semantic conversion.

PSRL ontology core modules include: form, function, behavior, products, assembly, specifications, objects, collections shape, features, materials and constraints. Among them, the specification refers to user needs and engineering information related to the content. Assembly refers to products collection. Products refers to the actual design entities, it has a certain behavior, function and form. Form contains material and geometry, etc. PSRL core relationship includes the parent-child relationship on the hierarchy and the relationship between objects. Using core relations and concepts, PRSL ontology by encoding the formation of a clear PSRL descript language.

The core of the semantic relations and the core concept is based on the characteristic model of standard expression. Simple concept further combined by superposition level as the new concept, so that for a typical set of generalized semantic CAX system modeling. On this basis, PSRL semantics to define a new relationship with the core concept, to ensure the body has sufficient semantic practicality and scalability. PSRL has gradually become the semantic information exchange in the middle of the language. Below is a part of PSRL semantic representation:

\section{Industry characteristics and application framework}

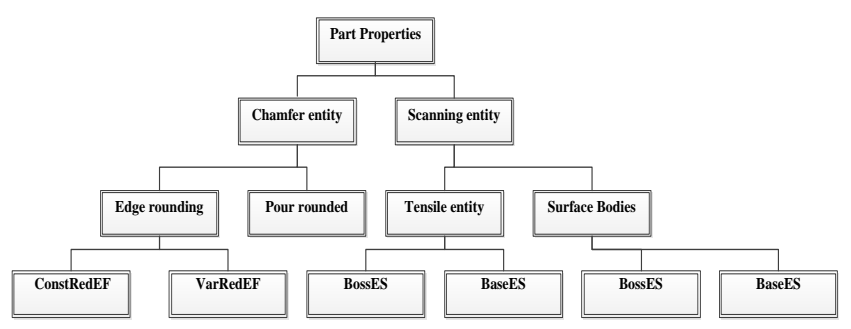

Fig1: PSRL semantic graph of parts

Mechanical engineering as a traditional engineering discipline, compared with other professions, has the following features:

a) Design and manufacture process of the products is a process of intensive and knowledge intensive experience. The process of its accumulated experience is particularly important. 
b) Product diversification, serialization, variety, focuses on technological innovation, upgrading faster way to manage the implementation of batch management products.

C) Production mode diversification, have large, medium and small batch production mode, including assembly technology as the core.

d) Around the customer order-based production methods.

E) Special equipment and general equipment arrangement as whole, specialized equipment closely related to the quality of the products, the use of special equipment and management have higher requirements.

F) Manufacturing process adopted by the method, equipment, processing technology, etc. are closely related to the production process has contact.

g) The higher the degree of information technology in the manufacturing process, the more likely the emergence of unbalanced data, the information cannot be re-used and shared retardation and other information.

In the mechanical engineering company, in order to reuse the knowledge management and management effectively, facilitate the information system of information sharing and interoperability between heterogeneous systems, according to the characteristics of the industry, refer Knowledge ontology application in other areas, and puts forward the following application framework (20-21).

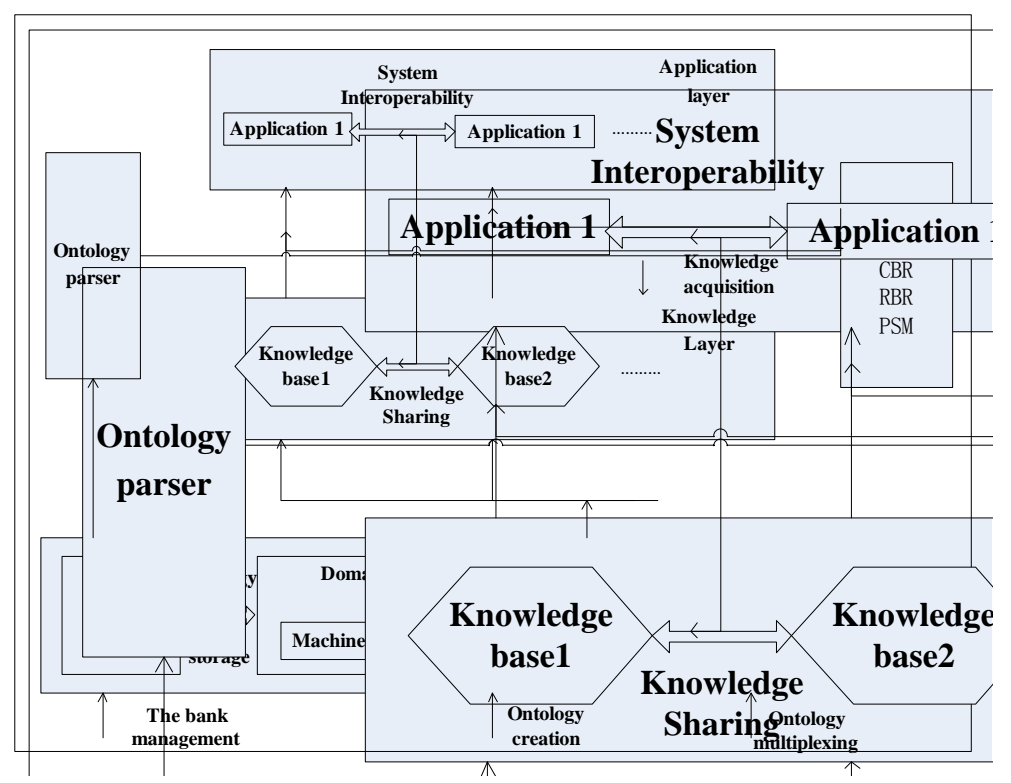

Fig2: Ontology Application Framework and Technology

Application layer can contain any knowledge of the system, such as: CAX systems, product configuration management, product modeling, collaborative manufacturing and collaborative product development. Mechanical engineering ontology in accordance with a certain proportion of particle size is established, such as: machining, process, tools, extra bed, business processes, product design, enterprise and workshop etc. establish the ontology in the field of machinery. According to the actual needs, establish more detailed granularity ontology through ontology integration and mapping of the ontology to form a fine-grained bulk coarse-grained. Coarse-grained ontology gradually forms knowledge base of application system, using the shared reasoning technology, and realized the application of knowledge.

\section{Applications and Limitations}

The purpose of research ontology is to establish knowledge-based systems of knowledge-based prototype, Ontology interoperability in heterogeneous and has a significant advantage in knowledge sharing, have a very broad application prospects in the mechanical field. Such as: product configuration management, product modeling, information exchange, methodology and so on. In 
practical applications, the ontology still has some limitations, for the expression of procedural knowledge, how to find effective problem-solving method, only through combined and procedural knowledge, to broaden the knowledge in the industry.

\section{References}

[1] FOX J,ALABASSI A,PATKAR V,et al. An ontological approach to modeling tasks and goals [J]. Computers in Biology and Medicine, 2006,36 (7-8): 837-856.

[2] KAVOURAS M, KOKLA M, TOMAI E. Comparing categories among geographic ontologies[J]. Computers \& Geosciences, 2005,31(2):145-154.

[3] YEH I, KARP P D, NOY N F, et al. Knowledge acquisition, consistency checking and concurrency control for gene ontology (GO) [J]. Bioinformatics, 2003, 19(2):241-248.

[4] USCHOLD M, GRUNINGER M. Ontologies:principles, methods and applications[J]. Knowledge Engineering Review, 1996, 11(2): 93-155.

[5] XU Y, LV L, REN W, et al. A semantic Web based model for product cooperative design[C] Proceedings of the 9th International Conference on Computer Supported Cooperative Work in Design. Berlin, Germany: Sptinger, 2005: 1177-1182.

[6] SUDARSAN R, FENVES SJ, SRIRAM R D, et al. A product information modeling framework for product lifecycle management [J]. Computer-Aided Design, 2005, 37(13): 1399-1411.

[7] LEE I, LEE S, LEE T, et al. Practical issues for building a product ontology system[C] Proceedings of the 2005 International Workshop in Data Engineering Issues in ECommerce(DEEC’05). Los Alamitos, Cal. USA: IEEE Computer Society, 2005:16-25.

[8] NECHES R, FIKERS R, FININ T, et al.Enabling technology for knowledge sharing [J]. AI Magazine, 1991, 12(3):36-56.

[9] STUDER T R, BENJAMINS V R, FENSEL D. Knowledge engineering: principles and methods [J]. Data Knowledge Engineering, 1998, 25 (12):161-197.

[10] GENESERETH M R, NILSSON N J. Logical foundations of artificial intelligence [M]. San Francisco, Cal. USA: Morgan Kaufmann Publishers, 1987: 9-13.

[11] STEVENS R, GOBLE C A, BECHHOFER S. Ontology based knowledge representation for bioinformatics[J]. Briefings in Bioinformatics,2000, 1(4):398-414.

[12] EHRIG M, SURE Y. Ontology mapping-an integrated approach[R]. Karlsruhe, Germany: University of Karlsruhe, 2004.

[13] USCHOLD M, KING M, ZORGIOS Y, et al. The enterprise ontology[R]. Edinburgh, UK: The University of Edinburgh, 1997.

[14] GUARINO N. Formal ontology, conceptual analysis and knowledge representation [J] . International Journal of Human-Computer Stuties, 1995, 43(5/6): 625-640.

[15] DEVEDZIC V. Understanding ontological engineering [J]. Communications of the ACM, 2002, 45(4): 136-144.

[16] VEGA J C A, GOMEZ-PEREZ A, TELLO A L, E et al. Agent: an ontology-bsaed WWW borker to select ontologies Proceedings of WorkShop on Application of Ontologies and PSMS. New York, N. Y. ,USA; John Wiley \& Sons ,1998 , 16-24.

[17] GRUNINGER M, FOX M S. Methodology for the design and evaluation of ontologies Proceedings of UCAI-95 Workshop on Basic Ontological Issues in Knowledege Sharing. Menlo Park, Cal, USA: AAAI Press, 1995.

[18] KNIGHT K, CHANDER I, HAINES M, et al. Filling knowledge gaps in a broad-coverage MT system[C] Proceedings of IJCAI-95. Menlo Park. , USA: AAAI Press, 1995: 1390-1397.

[19] DOMINGUE J, MOTTA E, GARCIA O C. Knowledge modeling in webonto and OCML; a user guide. http:// kmi.open. ac.uk/projects /webonto/user_guide, 2.4. pdf.

[20] KARP P D, CHAUDHRI V K, THOMERRE J. XOL: An XML-based ontology exchange language. (1999-08-31). http: // w w w .ai. sri.com /pkarp/xol/xol.html.

[21] XIA Yan, ZHANG Zhongping, CAO Shunliang, et al. Application of gene ontology in integrating bioinformatics data[J]. Computer Engineering, 2005, 31(2): 57-58, 76(in Chinese). 